\title{
EPILEPSY.
}

\author{
By W. RUSSELL BRAIN, D.M. (Oxon.), F.R.C.P. (Lond.).
}

(Physician, with Charge of Out-patients, London Hospital ; Physician, Hospital for Epilepsy and Paralysis, Maida Vale, and the Royal London Ophthalmic Hospital.).

\section{The Nature of Epilepsy.}

It is strange that in a generation so much occupied both with psychology and with scientific research little, if any, attention has been paid to the psychology of the discoverer. Without going deeply into this question it is clear that scientific discoveries are of different kinds. Some are the outcome of the patient accumulation of facts by many workers, chance largely determining by whom the copingstone is finally placed in position. The discovery of fundamental principles, however, seems more often to be the product of individual genius, of a certain kind of vision personal to the discoverer. The isolation of insulin, great achievement though it was, would probably have been effected by someone else at about the same time if Banting had never lived, but if Hughlings Jackson had not been born the development of neurology might well have been retarded by a generation. It is a paradox of fame, however, that the discoverer of a substantial thing, a bacillus, an extract or a disease, achieves a more certain immortality than the thinker who discovers a principle, for principles of permanent validity soon become part of current thought and in time appear so obvious as to seem to have needed no discoverer. Thus Hughlings Jackson, the founder of modern neurology, is hardly remembered outside the neurological world, except as the eponym of a localized convulsion, the recognition of which was in a sense a trivial by-product of his observations. It is worth while, therefore, to emphasize that to preface a modern article on epilepsy with an allusion to Hughlings Jackson is no mere act of commemorative piety. The whole framework of our present conception of epilepsy is Jacksonian, as is shown by the quotations in inverted commas in this article, which are from Jackson's writings.

"We have in each case of epilepsy three lines of investigation: Physiological, which deals with abnormal Function of nerve tissue; Anatomical, which deals with abnormalities of particular Structures (organs); and Pathological, which deals with abnormalities of Nutrition." Jackson's first great generalization was that at the physiological level "a convulsion is but a symptom, and implies only that there is an occasional, an excessive and a disorderly discharge of nerve tissue on muscles." This, no doubt, depends upon an abnormal and uncontrolled conversion of the potential energy of certain neurones into kinetic energy. The next step is to try "in each case where there is a paroxysmal presentation of symptoms to find the seat of the discharging lesion " corresponding to them. The effect of putting this anatomical question in respect of the large variety of convulsions was to lead Jackson to the recognition that many epileptic fits are focal in origin and the disturbance may remain limited to a localized area of one cerebral hemisphere. The "Jacksonian convulsion" characterized by its sharply focal onset and its slow and orderly spread corresponding to the representation of parts of the body in the precentral convolution is one such focal fit. Another is that now usually known as an uncinate fit, in which an "intellectual aura," called by Jackson a "dreamy state" is associated with an hallucination of smell or taste and frequently with " movements," chewing, tasting, spitting, 'implying' (?) an epileptic 
discharge beginning in some part of the gustatory centres." Foerster and others, as a result of electrical stimulation of the cerebral cortex in man, have not only increased our knowledge of the varieties of focal convulsion but also have shown how the site of origin of an epileptic discharge may determine an asymmetry of posture and involuntary movement in the head and limbs in a generalized fit. The aura of an epileptic attack is significant as probably indicating the anatomical situation at which the epileptic discharge begins.

In addition to the tonic and motor phenomena, consciousness is frequently impaired or lost in an epileptic attack. How, it may be asked, is a symptom of apparently so negative a character as loss of consciousness to be explained as a result of an excessive discharge of nerve tissue? "That loss of consciousness, a negative symptom, should be ascribed to an active process (discharge) seems, at first glance, unlikely. We see, however, that patients lose the use of an arm during the stage of tonic spasm of it as much as they do in paralysis of it. I think, then, that consciousness may be lost during the excessive discharge of the anatomical substrata of consciousness. Besides other reasons for ascribing loss of consciousness from an epileptic discharge, the discharge is rapid. Time is required for consciousness." It follows that loss of consciousness is no essential part of an epileptic attack. The discharge may be so limited in the hemisphere as not to involve the substrata of consciousness. The more widely the discharge spreads, the more likely is consciousness to be lost. On the other hand, a discharge of "the very highest nervous arrangements in the whole nervous system, and of those which have the greatest integration, that is to say, of the substrata of consciousness," is likely to be attended, as in petit mal, by loss of consciousness with little or no excitation of tonic contraction or movements.

Hughlings Jackson's interpretation of the physiology of epilepsy affords a ready explanation of the phenomena of the postepileptic state. The coma which follows a major attack is to be attributed to exhaustion of the substrata of consciousness following their excessive discharge, and is homologous with postepileptic hemiplegia following a severe localized convulsion involving one side of the body. The temporary presence of extensor plantar reflexes after an attack of grand mal is similarly an indication of exhaustion of the pyramidal fibres by the fit. In the disorders of conduct which sometimes follow epileptic attacks, especially petit mal, the mental automatism results "from over-action of lower nervous centres because the highest or controlling centres have been put out of use. . . . In other words there is (I) loss of control permitting (2) increased automatic action."

Hughlings Jackson's contribution to the Pathology of epilepsy, a term which he used in a somewhat special way, is perhaps of greater theoretical than practical importance. Briefly, he emphasized that in many cases of epilepsy there is no visible pathological change in the nervous system. The disturbance of function underlying the fit must therefore depend upon minute changes, which he thought might be the result of disturbance of Nutrition of the nerve cells. Even when a convulsion was the outcome of a gross lesion, such as a tumour of the brain, this could only operate indirectly, by causing instability of nervous tissue. He believed that " of nervous diseases of which the pathology is known, there are very few and possibly none in which the pathological change begins in the nervous elements of the nervous organ affected" and that this was true of epilepsy. 


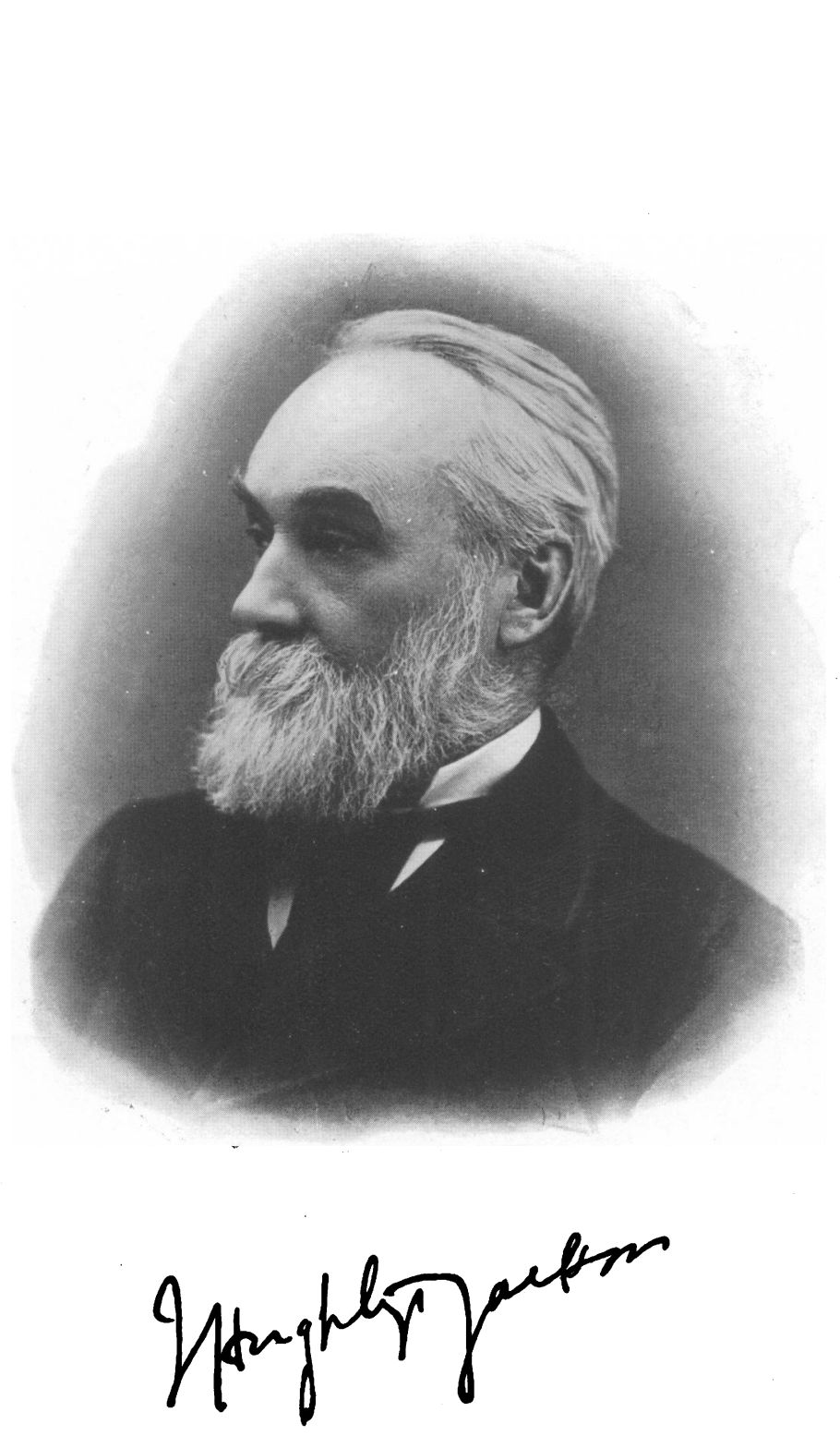




\section{The Causes of Convulsions.}

If we are to regard a convulsion as essentially an uncontrolled neural discharge depending upon the abnormally rapid conversion of the potential energy of the neurone into kinetic energy, it would seem to follow that this must possess a physico-chemical basis in a disturbed physico-chemical state of the neurone, the " minute change" of which Hughlings Jackson spoke. Inevitably, therefore, there are many causes of convulsions, since the underlying physico-chemical disturbance can be evoked directly or indirectly in many different ways. We can conveniently divide the known causes into local or general. Among the more important of the former are (I) conditions causing increased intracranial pressure ; (2) inflammatory conditions of the brain and its membranes-encephalitis and meningitis; (3) trauma; (4) congenital abnormalities, such as congenital diplegia and tuberose sclerosis; (5) degenerative states, such as cerebro-macular degeneration, the cerebral scleroses of infancy and the degenerations responsible for presenile dementia; (6) circulatory disturbances, such as cerebral arteriosclerosis and acute vascular lesions of the brain. General causes include (I) exogenous poisons, including the known convulsant drugs; (2) anoxæmia; (3) disordered metabolism, as in eclampsia, uræmia, hypoglycæmia, tetany and rickets; (4) acute infections in childhood.

Idiopathic epilepsy must be placed in a separate category. By some of Hughlings Jackson's followers his doctrine of the nature of epilepsy has been carried further than perhaps he himself would have allowed. The term "the epilepsies" has been applied to a large group of disorders characterised by convulsions, and it has been implied that the term "idiopathic epilepsy" is merely an expression of our ignorance as to the cause of the convulsions in certain cases. This conception is valuable in so far as it puts us on our guard against regarding a patient as suffering from idiopathic epilepsy without having made the fullest investigations to exclude the known causes of convulsions. Even when no such cause has been found it is wise to bear in mind that in course of time symptoms and signs may appear which show that an organic cause for the fits was present, though previously undetected. Nevertheless, the recognition that we may sometimes be mistaken in our diagnosis of idiopathic epilepsy does not prove that there is not a group of patients to whose disorder the name may be appropriately applied. The fact that in some 30 per cent. of cases of epilepsy there is a family history of convulsions indicates that there exists in such individuals a constitutional predisposition to the disorder which finds no expression in any visible lesion in the nervous system or elsewhere but doubtless involves a disturbance of " nutrition," the nature of which has yet to be discovered.

In considering the diagnosis of epilepsy the age of the patient is of paramount importance. In 75 per cent. of cases of idiopathic epilepsy the disorder first manifests itself before the age of 20 , and in 90 per cent. before the age of 30 . In childhood idiopathic epilepsy is far the commonest cause of convulsions, except perhaps in infancy, during which convulsions may occur as a symptom of rickets or as a reaction to an acute infection. Other causes of convulsions in childhood are rare. Epilepsy may be associated with infantile hemiplegia and congenital diplegia, and convulsions may occur during acute infections of the substance of the nervous system and of its coverings-acute encephalitis and meningitis. In the rare cerebral scleroses and degenerations of childhood fits, when they occur, are accompanied by progressive mental failure and paralysis. 
In adult life syphilis of the nervous system, especially general paralysis, and intracranial tumour are the commonest organic diseases of the nervous system leading to convulsions, until late middle age, when cerebral arteriosclerosis becomes the commonest cause. Convulsions may occur also as a result of acute vascular lesions of the brain-cerebral hæmorrhage, thrombosis and embolism-and among less frequent causes which should be borne in mind are uræmia, heart block, leading to Stokes-Adams attacks, and hypoglycæmia, occurring as the result of an excessive production of insulin by a tumour composed of the pancreatic islet cells.

\section{The Treatment of Epilepsy.}

No disorder better repays careful and discriminating treatment than epilepsy. In fact, the adequate care of an epileptic patient involves as much supervision and individualization as that of a diabetic. The unsatisfactory results so frequently obtained in the treatment of epilepsy are due to a lack of appreciation of this fact. It is first necessary to decide whether the patient can be treated at home, or whether institutional care is necessary. As a rule the latter is desirable in the case of patients having severe and frequent fits and of those in whom epilepsy is associated with marked mental defect. If the patient is to be treated at home he should lead as normal a life as possible. All epileptics are better when occupied, and if possible, therefore, arrangements should always be made for the child and adolescent to attend school and for the adult to have some form of occupation. Certain safeguards are, of course, necessary to avert danger in the event of a convulsion. Epileptics are now forbidden by law to drive a motor car, and riding a bicycle may be equally dangerous. Working at heights and in close association with machinery must also be forbidden and no epileptic should bathe in the sea or river unless accompanied by a strong swimmer who is aware of the danger. Nevertheless, it is surprising how few epileptics fall victims to the dangers which appear to beset them.

Most epileptic patients may be allowed an ordinary diet, but they are frequently voracious eaters and this tendency must be curbed and they should be forbidden to eat constipating or indigestible food. In some cases a special article of diet may be found to precipitate attacks and must then be avoided. Aperients are often necessary to prevent constipation. During recent years a ketogenic diet has been used in the treatment of epilepsy. A diet rich in fats and poor in carbohydrates is given, the ratio between fats and carbohydrates being increased until ketone bodies appear in the urine. On such a diet about 30 per cent. of epileptic children have become free from attacks and in a further 20 per cent. the frequency of the attacks is reduced. Adults are less responsive than children to this method of treatment. The diet requires to be accurately calculated and constant supervision is usually necessary in order to prevent the child from eating illicit sweets or other carbohydrate food. Benefit, when it occurs, lasts only as long as a child keeps to the diet, and this mode of treatment should be reserved for those patients who have failed to respond to other and simpler methods.

The use of drugs requires much judgment. The object of drug treatment is to abolish the fits for a sufficient length of time to enable the patient to lose the "epileptic habit." This must be explained to the patient and his relatives at the beginning of the treatment and they should be warned that drugs in some form are likely to be required for at least three years. Sporadic drug treatment for a 
few weeks at a time is probably worse than useless. Since the first object of treatment has not been achieved until the attacks have been completely suppressed, it is usually necessary during the first few months of treatment to vary the drugs given and their doses, until either this result has been achieved or it appears to be impossible of achievement. In administering drugs attention must be paid to the rhythm of the attacks. When they occur regularly at the same hour of the day or period of the month, the doses are timed so as to produce their maximal effect when the attack is expected. Thus, when the attacks are nocturnal or occur in the early morning, a single dose at bedtime may be sufficient. When they occur only at the menstrual periods medication can sometimes be confined to the week before and after the period. When the fits are irregular a dose must be taken two or three times a day. It is wise to begin treatment with bromide. There seems no advantage in using several different bromides concurrently. Potassium bromide should be given in doses of Io to I5 grains three times a day for an adult. If this does not control the attacks, phenobarbital (luminal or gardenal) should be added, beginning with a dose of $\frac{1}{2}$ grain night and morning, and increasing up to 3 grains a day. This drug is more effective in the treatment of major than of minor attacks. Most patients do best on bromide and luminal in combination. Some, however, do better on luminal alone. Calcium-dibrom-ethylbarbituric acid (superminal) is sometimes effective when both bromide and luminal fail. It may be given in doses of 0.5 of a gramme ( $7 \frac{1}{2}$ grains) in tablet form, from once to three times a day. As a rule it has no depressant effect upon the mental functions. It will sometimes be found that a combination of bromide, luminal and superminal will suppress the attacks when each of these drugs singly has failed. Tincture of belladonna in doses of from 5 to Io minims, and sodium biborate in doses of I5 to 30 grains, are useful as adjuvants to bromide in some cases, the former being sometimes especially effective in the treatment of petit mal.

Good results in the treatment of epilepsy have lately been claimed for Fitzsimon's Venene, a detoxicated snake venom. This, however, is not at present obtainable in this country.

Status epilepticus is most effectively treated by the subcutaneous injection of luminal, 5 grains of the soluble sodium salt (luminal sodium) being given subcutaneously in 25 per cent. solution to an adult, a correspondingly smaller dose being given to a child. The dose can be repeated if necessary. An alternative method of treatment is to administer sedative drugs by the rectum, 60 grains of chloral hydrate or sulphonal, or an ounce of paraldehyde, being given in this way. The withdrawal of cerebrospinal fluid by lumbar puncture is a useful adjuvant measure, and in every case the lower bowel should be well washed out by enemata, rectal salines being given if unconsciousness is prolonged.

The question of the advisability of surgical treatment is not uncommonly raised, and there has recently been a revival of interest in this, since Foerster and Penfield have advocated it in certain cases of traumatic epilepsy. It is necessary to emphasize that operation on the skull is likely to be advisable in only a very small proportion of patients with epilepsy, even when the disorder has been traumatic in origin. Surgery can only be effective when there is evidence that the cerebral cortex is being irritated by a scar uniting it to the dura mater, and the existence of such a lesion must be proved by X-ray examination of the skull following the introduction of air into the cerebral ventricles. 